\title{
BMJ Open Physical comorbidity and use of healthcare services in people with schizophrenia: protocol for a systematic review
}

\author{
Mercè Salvador Robert, ${ }^{1}$ Alejandro Porras-Segovia, ${ }^{2}$ Inmaculada Peñuelas-Calvo, ${ }^{3}$ \\ Enrique Baca-Garcia (D) 2,4,5,6,7,8
}

To cite: Salvador Robert M, Porras-Segovia A, PeñuelasCalvo I, et al. Physical comorbidity and use of healthcare services in people with schizophrenia: protocol for a systematic review. BMJ Open 2021;11:e053324. doi:10.1136/ bmjopen-2021-053324

- Prepublication history and additional supplemental materia for this paper are available online. To view these files, please visit the journal online (http://dx.doi.org/10.1136/ bmjopen-2021-053324).

Received 13 May 2021 Accepted 17 November 2021

Check for updates

(c) Author(s) (or their employer(s)) 2021. Re-use permitted under CC BY-NC. No commercial re-use. See rights and permissions. Published by BMJ.

For numbered affiliations see end of article.

Correspondence to

Professor Enrique Baca-Garcia; ebacgar2@yahoo.es

\section{ABSTRACT}

Introduction People with schizophrenia die about 15-20 years earlier than the general population. A constellation of factors contributes to this gap in life expectancy: side effects of psychotropic drugs, unhealthy lifestyles (inactivity, unhealthy diet) and inequality in the provision of healthcare services. This is a topic of main importance, which requires constant update and synthesis of the literature. The aim of this review is to explore the evidence of physical comorbidity and use of healthcare services in people with schizophrenia.

Methods and analysis We will conduct a systematic literature search in the databases PubMed/MEDLINE, EMBASE, Scopus, Web of Science, PsycINFO and Cochrane Library, Proquest Health Research Premium Collection, in order to identify studies that answer to our research question: Are patients with schizophrenia different from the non-psychiatric population in terms of physical comorbidity and use of healthcare services? Two authors will independently review the studies and extract the data. Ethics and dissemination This study does not include human or animal subjects. Thus, ethics considerations are not applicable. Dissemination plans include publications in peer-reviewed journals and discussion of results in psychiatric congresses.

PROSPERO registration number CRD42020139972.

\section{BACKGROUND}

About 7 out of every 1000 people will suffer from schizophrenia in their lifetime. ${ }^{1}$ There is an excess mortality in patients with schizophrenia, which is mainly the result of a higher prevalence of physical conditions. ${ }^{12}$ For instance, patients with schizophrenia are at two to five times higher risk of developing diabetes than the rest of the population. Schizophrenia has been described as a 'lifeshortening illness', and physical comorbidity accounts for $60 \%$ of premature deaths unrelated to suicide. ${ }^{4-6}$ Nearly $50 \%$ of patients with schizophrenia comorbid medical conditions, but these are often underdiagnosed..$^{7-9}$

Evidence suggests that people with schizophrenia have not experienced the same improvement in life expectancy as the general population in recent decades. The mortality

\section{Strengths and limitations of this study}

The systematic review is methodologically sound

- The limitations of this study are the expected heterogeneity of the results, which will preclude carrying out a meta-analysis.

- This heterogeneity is likely to be the result of methodological designs, diagnostic variations, different clinical settings and cultural differences, including health policies and medical professionals' attitudes in each country.

- Another limitation is that language of studies will be restricted to English, French and Spanish, meaning that evidence published in any other language will be missed.

gap between people with schizophrenia and the general population not only persists but may have increased. ${ }^{10-12}$ Furthermore, the physical health of people with schizophrenia may have deteriorated since the start of the pandemic in the early 2020s, according to some studies. ${ }^{13}$ Patients with schizophrenia are also less likely to be vaccinated against COVID-19. ${ }^{14}$

One of the factors that may be involved in the poorer physical health of patients with schizophrenia are the side effects of secondgeneration antipsychotics. ${ }^{15}$ There are also barriers to the provision of adequate healthcare and help-seeking in this population. These barriers may be related to the patient and their illness, to the attitudes of clinicians and to the structure of the healthcare system. Fragmented health systems, financial difficulties in accessing healthcare or patients' inability to describe their physical problems, are some of the factors that may explain the substandard medical care of patients with schizophrenia. ${ }^{16}$ General practitioners and specialists in fields other than psychiatry often feel insecure when treating patients with schizophrenia ${ }^{17}$ and may even fear them. ${ }^{18}$ Patients with schizophrenia may suffer from 
the stigma associated with mental health at the hands of health professionals, putting their physical health at risk. In a survey of patients with schizophrenia in 27 countries, $17 \%$ reported feeling discriminated against when being treated for their physical health problems. ${ }^{19}$ Psychiatrists, for their part, lack the training needed to take care of their patients' physical health, but are often the main healthcare provider of patients with schizophrenia, both for their mental and physical health problems. ${ }^{20}$ Further collaboration between departments and a more holistic approach are needed for taking care of the physical health of patients with schizophrenia.

There are other risk factors that can contribute to this excess mortality, such as lifestyle: studies show that people with schizophrenia have higher rates of smoking, unhealthy diet and sedentarism. ${ }^{21-24}$

There are some previous systematic reviews about different aspects of physical comorbidity in patients with schizophrenia. For instance, a review by Janssen et al, carried out in 2015, showed a high prevalence of medical conditions among people with schizophrenia. ${ }^{25}$ For their part, a meta-analysis by Vancampfort et al revealed an increased risk of metabolic syndrome in people with schizophrenia compared with the general population. ${ }^{26}$ Despite the great contribution of these and other previous reviews, there are still gaps of knowledge that deserve to be explored, such as the use of healthcare services among people with schizophrenia. Moreover, this is a crucial topic, which requires constant update of the literature. The aim of this review is to explore the prevalence of physical comorbidity and use of healthcare services among people with schizophrenia. Our research question is 'Are there quantitative differences patients regarding physical comorbidity and use of healthcare services between patients with schizophrenia and the non-psychiatric population?'

\section{METHODS AND ANALYSIS}

The review followed the Preferred Reporting Items for Systematic Reviews and Meta-Analyses Protocols (PRISMA) guidelines. ${ }^{27}$ PRISMA guidelines checklist is shown in table 1 .

\section{Inclusion/exclusion criteria}

Inclusion criteria are:

1. Original observational (cohort, case/control or crosssectional) studies published in peer-reviewed journals.

2. Studies that compare people with schizophrenia (diagnosis established either by a clinician or by using standardised questionnaires) with non-psychiatric populations (either clinical or non-clinical).

3. Studies that explore at least one of the following outcomes:

a. Quantitative differences in the prevalence and/or clinical features of physical conditions in schizophrenia patients versus non-psychiatric populations. b. Quantitative differences in use of healthcare services in schizophrenia patients versus non-psychiatric populations, including: bed occupancy, hospitalisation stays, wait times to surgery, adherence to treatment plans and admission to trials of new drugs.

Exclusion criteria are:

1. Case studies, case series and studies with $n=1$

2. Reviews

3. Clinical trials

There will be no restrictions regarding healthcare setting (inpatients, outpatients, community-dwelling people, etc) or treatment received (people with or without treatment).

There will be no restrictions regarding publication date of the studies.

Publication language will be restricted to English, Spanish or French.

Main outcomes are: Physical comorbidity (prevalence of physical conditions, clinical features and prognosis of such conditions); Use of healthcare services (hospitalisation days, outpatient appointments, emergency visits, expenses).

\section{Search strategy}

We will conduct a systematic literature search of the following databases: PubMed/MEDLINE, EMBASE, Scopus, Web of Science, PsycINFO and Cochrane Library, Proquest Health Research Premium Collection.

Language restriction: English, Spanish or French.

There will be no restrictions by date.

The following search terms will be used: "Schizophrenia" [Mesh]) AND "Comorbidity"[Mesh])) AND "schizophrenia"[Title]) AND (("medical comorbidity") OR "physical comorbidity")

The references of included studies will also be screened. Full search strategy is shown in online supplemental file 1.

Planned start date is 20 November 2021. Planned completion date is 20 February 2022.

\section{Study selection and data extraction}

Titles and/or abstracts of the paper retrieved will be screened independently by two reviewers to identify studies that potentially meet the inclusion criteria. The full text of these studies will be independently assessed by the two reviewers. Discrepancies between reviewers will be resolved by discussion, with the participation, if necessary, of a collaborator.

Data will be identified, checked and mined by two independent reviewers. The following variables will be collected: author; design; country; year of study publication; study design; sample size; age of the sample; gender distribution of the sample; clinical setting-inpatients/outpatients-; outcomes; measures; main findings. Authors of the selected studies will be contacted if additional information is needed. A qualitative synthesis of data will be performed. The strength of the body of evidence regarding our research topic will be assessed 
Table 1 Preferred Reporting Items for Systematic Reviews and Meta-Analyses Protocols 2015 checklist: recommended items to address in a systematic review protocol

\begin{tabular}{llll}
\hline Section and topic & Item no & Cheklist item & Page no \\
\hline Administrative information & & & \\
Title: & $1 \mathrm{a}$ & Identify the report as a protocol of a systematic review & 1 \\
$\quad$ Identification & $1 \mathrm{~b}$ & If the protocol is for an update of a previous systematic review, identify as such \\
$\quad$ Update & 2 & If registered, provide the name of the registry (such as PROSPERO) and registration number 6 & N/A \\
Registration & & &
\end{tabular}

Authors:

\begin{tabular}{|c|c|c|c|}
\hline Contact & $3 a$ & $\begin{array}{l}\text { Provide name, institutional affiliation, e-mail address of all protocol authors; provide physical } \\
\text { mailing address of corresponding author }\end{array}$ & 2 \\
\hline Contributions & $3 b$ & Describe contributions of protocol authors and identify the guarantor of the review & 10 \\
\hline Amendments & 4 & $\begin{array}{l}\text { If the protocol represents an amendment of a previously completed or published protocol, } \\
\text { identify as such and list changes; otherwise, state plan for documenting important protocol } \\
\text { amendments }\end{array}$ & N/A \\
\hline
\end{tabular}

Support:

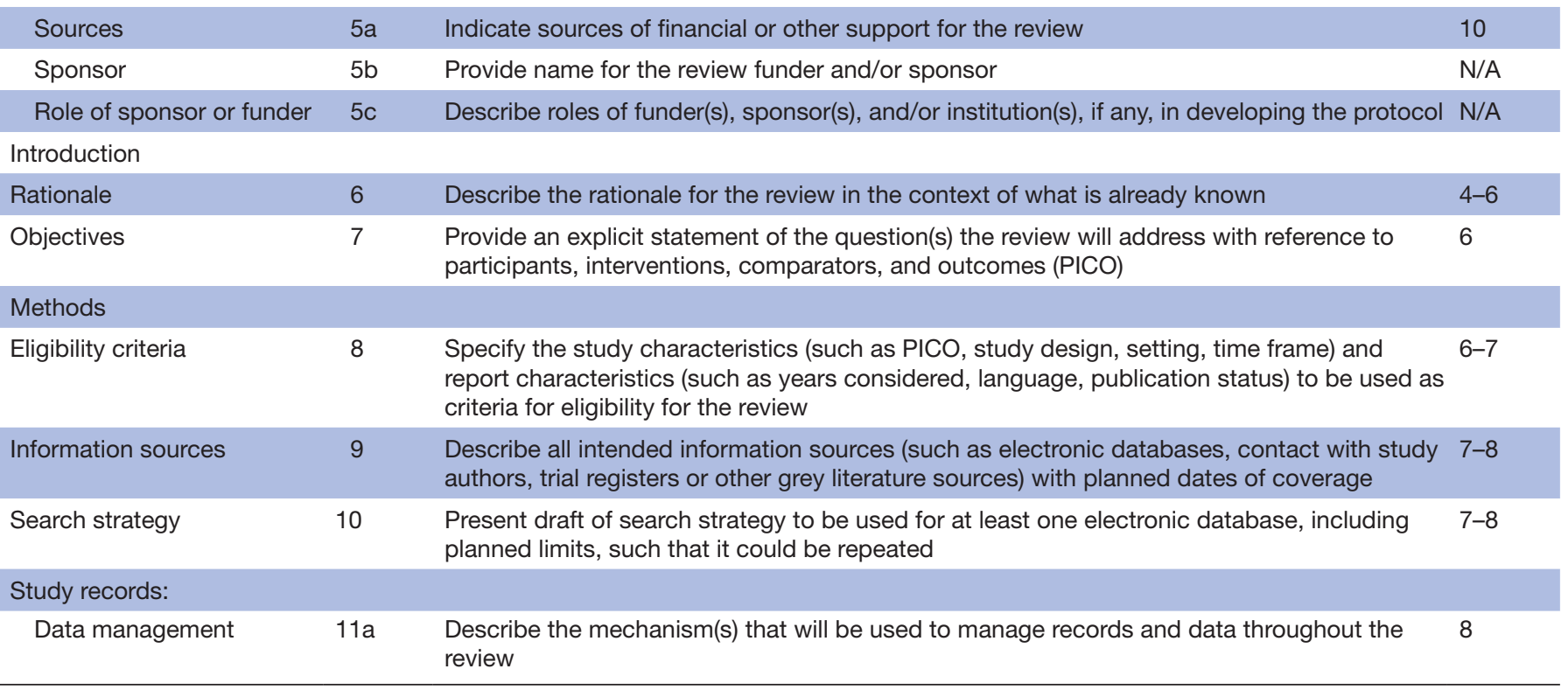

N/A, not available; PROSPERO, International Prospective Register of Systematic Reviews.

through the Grading of Recommendations Assessment, Development and Evaluation system. ${ }^{28}$

\section{Risk of bias (quality) assessment}

All eligible studies will be reviewed and critically appraised. Quality will be independently assessed by two reviewers. Discrepancies between reviewers will be resolved by discussion, with the participation, if necessary, of a collaborator. Aspects assessed will include risk of bias, methodological design, quality of reporting, etc.

We will use the Newcastle-Ottawa Scale to assess the quality of case control and longitudinal cohort studies. ${ }^{29}$ Strengthening the Reporting of Observational Studies in Epidemiology individual component checklists will also be used to appraise the studies. ${ }^{30}$ Studies will be considered methodologically solid if they present an appropriate design, their case and control groups are comparable, and they are free of selection bias, attrition bias, detection bias, and reporting bias.

\section{Patient and public involvement}

It was not possible to involve patients, families, healthcare professionals or other members of the community in the design, conduct, reporting, and dissemination plans of our research.

\section{DISCUSSION}

Regarding our dissemination plans, this systematic review will be published in a peer-reviewed journal. This systematic review presents with some potential limitations: language of articles will be restricted to English, French or Spanish, meaning that evidence published in any other language will be missed. The expected heterogeneity of the articles to be reviewed will most likely preclude a quantitative synthesis of results. Finally, the broad topic may represent a challenge but will contribute to closing gaps of knowledge in the existing literature. 
Increasing knowledge about physical comorbidity and use of healthcare services in patients with schizophrenia will contribute to informing programmes aimed at providing medical care of this population. This, in turn, is expected to reduce their excess mortality and increase their quality of life, as well as reduce costs by avoiding medical complications.

\section{Ethics and dissemination}

This study does not include human or animal subjects. Thus, ethics considerations are not applicable. Dissemination plans include publications in peer-reviewed journals and discussion of results in psychiatric congresses.

\section{Author affiliations}

${ }^{1}$ Department of Psychiatry, Hospital Universitario de Mostoles, Mostoles, Spain

${ }^{2}$ Instituto de Investigacion Sanitaria de la Fundacion Jimenez Diaz, Madrid, Spain

${ }^{3}$ Psychiatry, Hospital 12 de Octubre, Madrid, Spain

${ }^{4}$ Department of Psychiatry, Hospital Universitario Fundacion Jimenez Diaz, Madrid, Spain

${ }^{5}$ Department of Psychiatry, Madrid Autonomous University, Madrid, Spain

${ }^{6}$ CIBERSAM (Centro de Investigacion en Salud Mental), Carlos III Institute of Health, Madrid, Spain

${ }^{7}$ Universidad Catolica del Maule, Talca, Chile

${ }^{8}$ Department of psychiatry, Centre Hospitalier Universitaire de Nîmes, France, Nîmes, France

Contributors MSR, EB-G and AP-S designed the systematic review protocol. MSR and AP-S registered the review protocol in the PROSPERO database. AP-S and IP-C drafted the manuscript. All authors contributed substantially to the revision of the manuscript. All authors read and approved the final manuscript.

Funding This study received grant support from Instituto de Salud Carlos III (CM19/00026).

Competing interests None declared.

Patient consent for publication Not applicable.

Provenance and peer review Not commissioned; externally peer reviewed.

Supplemental material This content has been supplied by the author(s). It has not been vetted by BMJ Publishing Group Limited (BMJ) and may not have been peer-reviewed. Any opinions or recommendations discussed are solely those of the author(s) and are not endorsed by BMJ. BMJ disclaims all liability and responsibility arising from any reliance placed on the content. Where the content includes any translated material, BMJ does not warrant the accuracy and reliability of the translations (including but not limited to local regulations, clinical guidelines, terminology, drug names and drug dosages), and is not responsible for any error and/or omissions arising from translation and adaptation or otherwise.

Open access This is an open access article distributed in accordance with the Creative Commons Attribution Non Commercial (CC BY-NC 4.0) license, which permits others to distribute, remix, adapt, build upon this work non-commercially, and license their derivative works on different terms, provided the original work is properly cited, appropriate credit is given, any changes made indicated, and the use is non-commercial. See: http://creativecommons.org/licenses/by-nc/4.0/.

\section{ORCID iD}

Enrique Baca-Garcia http://orcid.org/0000-0002-6963-6555

\section{REFERENCES}

1 McGrath J, Saha S, Chant D, et al. Schizophrenia: a Concise overview of incidence, prevalence, and mortality. Epidemiol Rev 2008;30:67-76.

2 Laursen TM, Nordentoft M, Mortensen PB. Excess early mortality in schizophrenia. Annu Rev Clin Psychol 2014;10:425-48.

3 Suvisaari J, Keinänen J, Eskelinen S, et al. Diabetes and schizophrenia. Curr Diab Rep 2016;16:16.

4 Schoepf D, Uppal H, Potluri R, et al. Physical comorbidity and its relevance on mortality in schizophrenia: a naturalistic 12-year follow- up in general Hospital admissions. Eur Arch Psychiatry Clin Neurosci 2014;264:3-28.

5 Hjorthøj C, Stürup AE, McGrath JJ, et al. Years of potential life lost and life expectancy in schizophrenia: a systematic review and metaanalysis. Lancet Psychiatry 2017;4:295-301.

6 Lambert TJR, Velakoulis D, Pantelis C. Medical comorbidity in schizophrenia. Med J Aust 2003;178:S67.

7 Laursen TM, Munk-Olsen T, Gasse C. Chronic somatic comorbidity and excess mortality due to natural causes in persons with schizophrenia or bipolar affective disorder. PLoS One 2011;6:e24597.

8 Goldman LS. Medical illness in patients with schizophrenia. J Clin Psychiatry 1999;60(Suppl 21):10-15.

9 Oud MJT, Meyboom-de Jong B. Somatic diseases in patients with schizophrenia in general practice: their prevalence and health care. BMC Fam Pract 2009;10:32.

10 Laursen TM, Nordentoft M, Mortensen PB. Excess early mortality in schizophrenia. Annu Rev Clin Psychol 2014;10:425-48.

11 Hayes JF, Marston L, Walters K, et al. Mortality gap for people with bipolar disorder and schizophrenia: UK-based cohort study 20002014. Br J Psychiatry 2017;211:175-81.

12 Siddiqi N, Doran T, Prady SL, et al. Closing the mortality gap for severe mental illness: are we going in the right direction? $\mathrm{Br} J$ Psychiatry 2017;211:130-1.

13 Kozloff N, Mulsant BH, Stergiopoulos V, et al. The COVID-19 global pandemic: implications for people with schizophrenia and related disorders. Schizophr Bull 2020;46:752-7.

14 Tzur Bitan D, Kridin K, Cohen AD, et al. COVID-19 hospitalisation, mortality, vaccination, and postvaccination trends among people with schizophrenia in Israel: a longitudinal cohort study. Lancet Psychiatry 2021;8:901-8.

15 Siafis S, Tzachanis D, Samara M, et al. Antipsychotic drugs: from receptor-binding profiles to metabolic side effects. Curr Neuropharmacol 2018;16:1210-23.

16 Muir-Cochrane E. Medical co-morbidity risk factors and barriers to care for people with schizophrenia. J Psychiatr Ment Health Nurs 2006;13:447-52.

17 Simon AE, Lauber C, Ludewig K, et al. General practitioners and schizophrenia: results from a Swiss survey. Br J Psychiatry 2005;187:274-81.

18 Magliano L, Punzo R, Strino A, et al. General practitioners' beliefs about people with schizophrenia and whether they should be subject to discriminatory treatment when in medical Hospital: the mediating role of dangerousness perception. Am J Orthopsychiatry 2017:87:559-66.

19 Harangozo J, Reneses B, Brohan E, et al. Stigma and discrimination against people with schizophrenia related to medical services. Int $J$ Soc Psychiatry 2014:60:359-66.

20 Oosthuizen P, Carey P, Emsley RA. Psychiatric disorders and general medical conditions: implications for the clinician. Afr J Psychiatry 2008;11:18-22.

21 Smith PH, Mazure CM, McKee SA. Smoking and mental illness in the U.S. population. Tob Control 2014;23:e147-53.

22 Vancampfort D, Firth J, Schuch FB, et al. Sedentary behavior and physical activity levels in people with schizophrenia, bipolar disorder and major depressive disorder: a global systematic review and metaanalysis. World Psychiatry 2017;16:308-15

23 Costa R, Teasdale S, Abreu S, et al. Dietary intake, adherence to Mediterranean diet and Lifestyle-Related factors in people with schizophrenia. Issues Ment Health Nurs 2019;40:851-60.

24 Bueno-Antequera J, Munguía-Izquierdo D. Exercise and schizophrenia. Adv Exp Med Biol 2020;1228:317-32.

25 Janssen EM, McGinty EE, Azrin ST, et al. Review of the evidence: prevalence of medical conditions in the United States population with serious mental illness. Gen Hosp Psychiatry 2015;37:199-222.

26 Vancampfort D, Stubbs B, Mitchell AJ, et al. Risk of metabolic syndrome and its components in people with schizophrenia and related psychotic disorders, bipolar disorder and major depressive disorder: a systematic review and meta-analysis. World Psychiatry 2015;14:339-47.

27 Shamseer L, Moher D, Clarke M, et al. Preferred reporting items for systematic review and meta-analysis protocols (PRISMA-P) 2015: elaboration and explanation. BMJ 2015;349:97647.

28 Foroutan F, Guyatt G, Zuk V, et al. Grade guidelines 28: use of grade for the assessment of evidence about prognostic factors: rating certainty in identification of groups of patients with different absolute risks. J Clin Epidemiol 2020;121:62-70.

29 Higgins JP, Green S. Cochrane Handbook for systematic reviews of interventions. The Cochrane Collaboration, 2011.

30 STROBE. Checklists - STROBE. Strobe-statement.org, 2021. Available: https://www.strobe-statement.org/checklists/ [Accessed 12 Oct 2021]. 